\title{
Fiber Optic Network Technology of Communication of Specialists via Mental Neurointerfaces
}

\author{
Evgeniy Bryndin ${ }^{1}$ \\ ${ }^{1}$ Research Centre "NATURAL INFORMATIC", Technological Platform FUTURE MEDICINE, Novosibirsk, \\ Russia \\ Correspondence: Evgeniy Bryndin, Research Centre "NATURAL INFORMATIC", Technological Platform \\ FUTURE MEDICINE, Novosibirsk, Russia. E-mail: bryndin15@yandex.ru
}

\author{
Received: August 10, 2021 Accepted: December 22, 2021 Online Published: December 30, 2021 \\ doi:10.5539/nct.v6n2p1 URL: https://doi.org/10.5539/nct.v6n2p1
}

\begin{abstract}
Resonance communication of specialists can be carried out at a distance in real time in dialogue mode through a mental neurointerface with two-way communication. Mental neurointerface on the principles of magnetic resonance tomography captures a hologram of brain activity of the internal speech of the inductor specialist and transmits it to the mental neurointerface of the recipient's interlocutor through ultra-sensitive multi section nano resonators waveguides. The mental neurointerface of the recipient's interlocutor perceives the transmitted hologram of the brain activity of internal speech and resonates its internal speech to it. An interlocutor of the recipient with equivalent semantic memory in a resonant way makes sense of the internal speech of the inductor specialist. He forms the response with internal speech and transmits it to the interlocutor with his mental neurointerface through nano resonators in the form of holograms of the brain activity of internal speech. Interlocutors, as specialists in one subject area, have a similar semantic memory. Semantic memories are considered similar if they correspond to the principle of gold section according to a professional thesaurus. Specialists and interlocutors must learn a professional thesaurus before starting a dialogue through mental neurointerfaces. Thus, the problem of transmitting and reading thoughts at a distance using high technology is solved, taking into account the psychological aspects of the interlocutors. The development of mental neurointerfaces and ultra-sensitive multi section nano waveguide resonators for transmitting holograms of internal speech brain activity is just beginning. The use of resonant communication by mental neurointerfaces through nano waveguides resonators waveguides is currently very relevant in many areas of life activity.
\end{abstract}

Keywords: resonance communication, mental neurointerface, nano waveguides resonators, internal speech.

\section{Introduction}

In 2011, the American company NeuroSky launched the brain-computer interface NeuroSky MindWave, a portable, wearable neurointerface affordable, on the world market. NeurosSkyproduces two types of neuro headsets: NeuroSky Mindwave and NeuroSky MindwaveMobile for Brain Fitness based on the HOA (Home of Attention) mental training program.

Scientists at Carnegie Mellon University in Pittsburgh (USA) collected different MRI research data and developed an algorithm for deep learning of the neural network for their analysis. The neural network interprets brain signals and builds a chain of its activities during internal speech. Scientists uploaded data on reactions and their causes into the algorithm, and at the exit they obtained a catalog of 240 brain reactions to individual people, places or physical actions. The neural network has learned to predict what a person thinks, according to MRI readings. The prediction accuracy was $87 \%$. The algorithm performed operations in the opposite order - when it received data on human action, it created an MRI image.

At Kyoto University, a team of scientists trained artificial intelligence to visualize thoughts from MRI data. The Japanese approached the study with the same idea as the Carnegie Mellon University team. For 10 months, they showed three subjects photographs of people and animals, figures and letters. During this time, the subjects developed a stable reaction to objects. During the display of the pictures, scientists shot indicators of brain activity using MRI. Later, the subjects were asked to think about these pictures, also shooting indicators. The researchers collected all the data and trained a neural network on them, which learned to create letters and even pictures based 
on the results of MRI, which are submitted to it for entry. Playback accuracy is quite high. Some image images are similar to a set of pixels, and some are like a slightly blurred photo of the original picture.

At the University of California, San Francisco, scientists have developed a neuroimplant and software that translates human brain activity into words and sentences. The implant is introduced into the brain, into the speech departments. The device captures brain activity and transfers it to the program. The output is synthesized speech. To simulate speech, implant developers investigated brain activity of dozens of subjects and compared data with movements of the vocal tract during this study. The result is a virtual model of each patient's speech apparatus. Simulated movements translated into sounds. The neural network was responsible for data analysis and speech synthesis. It was she who deciphered the brain commands and transformed them into a voice. The researchers used two machine learning algorithms. The first is a decoder that converts brain activity patterns generated during speech into virtual voice path movements. The second is a synthesizer that turns these movements into synthetic.

Internal speech recognition devices and software form together Brain-Computer Interface (BCI) technology neurointerfaces. Through $\mathrm{BCI}$, the brain and electronic devices exchange information. The technology for translating brain activity into speech is real. Internal speech can be controlled through a computer by manipulators and write individual words to those who are deprived of the ability to speak, through BCI. There are results in this direction, but not for the mass use of technology.

Elon Musk announced tests of Neuralink thought reading chips injected into the human brain in 2021. A non-standard Neuralink device, the purpose of which is based on communication between people and computers without using additional peripherals. A thought chip may be ready for human trials by the end of this year. One of the main tasks is to increase the capabilities of a person as a whole organism and the functions of the psyche: memories, reactions and others.

Mark Zuckerberg is developing technology that will allow people to read each other's thoughts and communicate using brainwaves can be a reality. Created by Facebook last year, the Building 8 research division is working to develop a new technology that is very reminiscent of reading thoughts. Facebook's Building 8 group is developing a hardware project using neuroimaging and electrophysiological data, which will lead to the creation of the so-called "communication platform of the future.

Elon Musk and Mark Zuckerberg are working on the secret projects of Neuralink and Building 8, respectively. The problem is that their development is based on invasive intervention, brain surgery will be required in the future. This makes this future too distant. It will take a long time to deploy technology that people willingly inject into their bodies through brain surgery.

Scientists are trying to figure out exactly where a person's thought is born and which part of the brain is responsible for this. One theory suggests that sensations and thoughts appear between the cortex of the brain and the inner surface of the skull, where there is brain fluid. The author of this hypothesis, Russian neurologist Andrei Bryukhovetsky, also suggested that the brain biofield emits special microwaves that are measurable. We managed to capture weak signals from all parts of the brain that are washed by brain fluid. These are temporal, occipital, parietal, right and left frontal lobes. That is, get confirmation that the brain really emits internal microwaves. Andrei Bryukhovetsky hopes for microwave transmission of thoughts over the distance.

The article discusses the resonance technology of communication of specialists through mental neurointerfaces with two-way communication through ultra-sensitive multisection nano resonators waveguides. The proposed technology of transmitting thoughts at a distance will change our lives.

\section{Functions of Mental Neurointerfaces}

Mental neurointerfaces are needed for dialogue with thoughts in real time at a distance through fiber optic communication, using multi-channel wave nano resonators, based on the fixation and transmission of a magnetic resonance hologram of internal speech. Modern technologies allow you to start creating mental neurointerfaces.

\subsection{Magnetic Resonance Fixation of Internal Speech by Mental Neurointerface}

Magnetic resonance imaging (MRI) today is used not only for diagnosis, but also for mapping the functional state of neural networks, allowing you to literally see the brain's work in real time (Ilya \& Alexandra, 2020; Liu, Yan, Li, Gao, \& Ding, 2020; Asadi, Wang, Olson, \& Obradovic, 2020; Wang, X. et al., 2020; Mitsuhashi et al., 2021).

The ability to directly observe the dynamics of cognitive (cognitive) brain activity, internal speech appeared only with the introduction of functional magnetic resonance imaging technology into research practice.

A little more than two decades ago, employees of the American research organization AT \& T Bell Laboratories described the principle of visualizing the activity of brain zones in real time using magnetic resonance imaging 
(MRI). A dynamic study of active zones of brain structures at the time of their activity was first tested on a person two years after the first publication.

MRI (magnetic resonance imaging) is a diagnostic procedure based on the effect of nuclear magnetic resonance. Modern MRI techniques allow not only to visualize organs with high quality, but also to investigate their function. Due to the absence of ionizing radiation, this method can be used without restrictions and repeated studies are repeatedly carried out.

It follows that the relative magnitude of the MRI signal can serve as a measure of the activity of brain zones. Moreover, the results obtained under the control of electroencephalography on the visual cortex of the primate open brain suggest that the MRI signal is a linear response to the electrical activity generated by the active neural ensemble.

Functional MRI focused on BOLD effect detection is today the optimal tool for mapping neuronal activity, more precisely, the functional state of neural networks - the basis for visualizing our thoughts and ideas. Using FMRT, you can literally see how our brains activate internal speech in real time.

The neurobiological technology of the "brain-computer interface" is closely associated with FMRT technology. We are talking about the possibility with the help of an electroencephalogram to obtain a display of a stable hologram of the bioelectric activity of the brain, tying it to the function of brain structures and the formation of new stable neural ensembles in them. The electroencephalogram is a source of information on intracerebral events. At the same time, FMRI makes it possible to visualize the real temporal and spatial dynamics of the brain. If you briefly describe the most general route of activation of brain structures during internal speech, then after the start, the broad cortical fields of the brain are first involved in the work, and such a "cognitive route" in the cerebellum ends.

Psychology is one of the most promising areas of use of neuroimaging technology by FMRT, because this scientific field is practically devoid of ideas about the localization (in the anatomical sense) of cognitive functions.

In one of the works of American researchers, an attempt was made to answer the question of the localization of brain structures designed to classify such cognitive categories as equality and efficiency. It turned out that the emotional assessment of the "effectiveness," "justice" and "general benefit" of the decision is carried out by three different brain structures. The brain department, called the "shell" (lat. Putamen), is responsible for efficiency, the bark of the "islet" (lat. Insula) protects the interests of justice, a cumulative measure of efficiency and inequality, that is, utility, assesses the septal organ (lat. Septum), while other areas of the brain are involved in the cognitive process.

The number of publications devoted to various fundamental and applied aspects of functional magnetic resonance imaging and the problems of the brain-computer interface has been steadily growing in recent years (mainly abroad, there are practically no domestic works on this list). The development of relevant technologies opens several promising application areas at once.

The development of cognitology, a direction of neurosciences that studies the basic mechanisms of the brain's work: "mental strategies," their localization, dynamics, ways of using and improving in everyday life, also opens up great prospects. The so-called "interactive stimulation" makes it possible to organize educational (therapeutic) feedback directly through the "interested" brain structure. By visualizing the cingulate gyrus or hippocampus, you get a chance of "direct conversation" with the brain.

When a person intentionally pronounces something to himself, the lower frontal gyrus (Brock's area) is active. Internal speech is associated with the state of passive brain work. The neural network of the passive mode includes the medial prefrontal cortex, the lateral parietal cortex, the posterior part of the cingulate gyrus and the entorhinal cortex (Figure 1). 


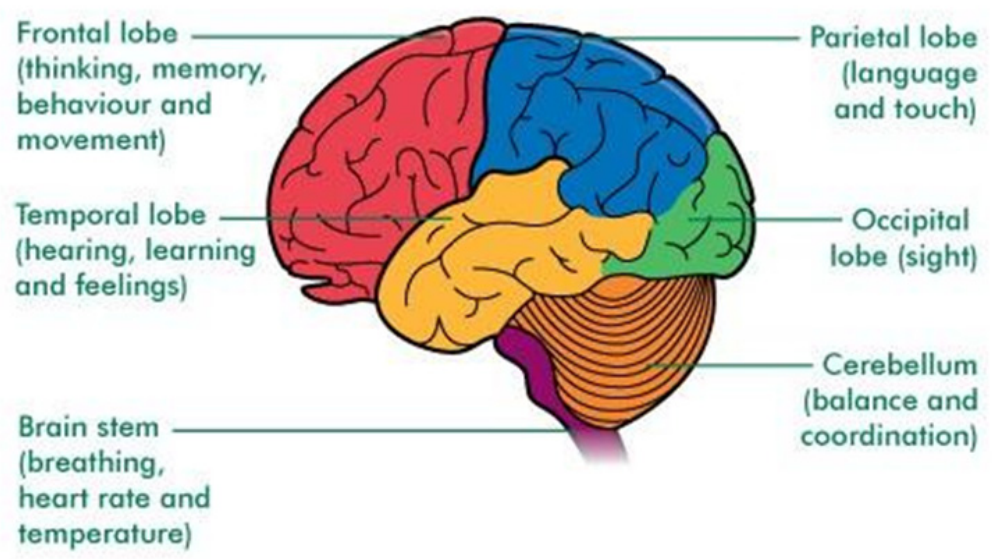

Figure 1. Brain functions.

Knowing how the brain is activated, it is possible to restore a person's internal speech through a resonant energy trace. Using non-invasive nanotissue that absorbs the brain activity of internal speech, it is possible to transfer this activity through mental neurointerface to another person (Bryndin, 2018; 2021).

Then it becomes possible to exchange the thoughts of people with similar semantic memory, based on the resonance of equivalent brain activities of thinking. Semantic memories are considered similar if they correspond to the principle of gold section according to a professional thesaurus, according to spherodynamics. Spherodynamics is a scientific discipline whose goal is to master the method of volume thinking. The simplest logical and mathematical constructions make it possible to clearly assess the principle of Unity of the World, perfectly known even in ancient times, but practically ignored by most modern scientists.

Formula: $\mathbf{F}=\mathbf{A}^{(2 / 3) 6}$ is sensor code that exhibits the seven-dimensional structure of the human sensory space, where $\mathrm{A}=240.2402$ is the universal World Constant, $\mathrm{F}=1,6180 \ldots$. - "golden proportion." At the same time, seven subspaces are resonantly interconnected, and the excitation of one of them, for example, by proper sound extraction, automatically excites the rest. And this is Unity with the whole World.

Semantic memory is memory for words, concepts, rules and abstract ideas; it is necessary to use the language. According to Tulving: "This is a mental thesaurus that organizes a person's knowledge of words and other verbal symbols, their meanings and references, about the connections between them and about the rules, formulas and algorithms for manipulating these symbols, concepts and relationships." Semantic memory has a much larger number of storage options, a much larger number of ways to establish relationships between concepts than figurative memory. Semantic memory is a very flexible, reconstructive, associative, and multimodal system. This is crucial in investigating questions regarding cognitive and neural dependencies in supporting cognition more broadly. Words have a high degree of imagination and specificity and are presented energetically with the help of sensory organs. Semantic wealth is a reflection of how many definitions and situations a word or concept is associated with.

Significant advances have appeared in experimental methods about the role of the hippocampus in semantic memory. Hippocam makes a solid contribution to maintaining semantic memory. Research has deepened the understanding of how words, concepts and meanings, as well as episodes and events, are created and preserved in memory and provide a new understanding of our two most typical human abilities: memory and language. Semantic memory in its entirety rests on the hippocampus, but still goes beyond it. In fact, there is significant neuroanatomical overlap (duplication) in the semantic network and the network of the passive mode of operation of the brain. The role of the hippocampus in tracking and determining the relationship between words in semantic memory in a manner similar to how the hippocampus tracks and determines the location in the surrounding space. The hippocampus promotes the tracking and construction of semantic associations between words. Scientists claim that the hippocampus plays a role in the prognostic processing of the language. HFB hippocampal power patterns were compared to each other based on semantic similarities calculated using LSA. The results showed that 
the pre-activation patterns of HFB activity were similar for images that were closer in semantic value to each other (Duff et al., 2020).

Neurophysiologist, Stanford University professor Karl Pribram, based on holographic theory, explained many other phenomenal brain-related phenomena. Memory, as one of the central functions of the brain, has a distributed rather than localized character, and each part of the brain can contain an integer just as a piece of holographic film contains information on which an entire image is created. The holographic theory explains how the brain manages to store a huge amount of information in a small space. Each biological structure, starting from the cell level, is the source of a wide range of fields. All vibrations or vibrations of internal organs are coherent. It is coherent radiation that creates a holographic image. In biological organism coherent fields form dynamic space-time interference structure-hologram on the basis of reference radiation. The radiation of each point of the object is as a reference relative to all other points.

Only the holographic structure of the brain allows us to explain how information is stored in human memory during clinical death, when the physical body (and the brain in particular) does not function, the potential difference of brain cells is zero. Information, if stored in brain cells, must be erased. And this does not happen, and a person after intensive care still instantly recognizes his relatives and friends, friends and just acquaintances. The holographic model explains the fact of instant recognition. A person, seeing a familiar face, immediately recognizes him. The wave principle of holography allows you to almost instantly extract from the vault the information that is encoded using the wave process.

Russian scientist, academician P.P. Garyaev, creator of a new science - wave genetics - in his book "Wave Genome" notes: human memory has a distinctly pronounced and well-studied holographic nature.

Academician Treasurer writes: "Today, a paradigm begins to emerge that proclaims that our brain is a hologram, and what we feel and see is a holographic virtual process.

The formation of a multicellular organism is associated with an evolutionary pattern unknown to us, in which each specialized cell, combining into a multicellular structure with other specialized cells, must find the corresponding interaction of holographic fields and the combination of holographic space and time inherent in each cell. The organism is an innumerable combination of various self-developing evolving holographic spaces, fields, and formations. It becomes clear why our brains can hold up to 10 billion bits of different signals in memory. Apparently, the amount of this knowledge is even greater, and the cells of sensory systems, which would seem to have only touch, sense of smell, hearing and vision, respond to other factors with holographic signs.

Berkeley neurophysiologists Russell and Karen Devalua made the crucial discovery. They transformed black and white cells into simple wave forms by the Fourier method. Devalua then conducted experiments to find out how brain cells in the visual part of the cerebral cortex respond to these new wave forms. The result shocked the scientific world: brain cells did not respond to the original images (black and white cells), but to the wave forms of these images. The discovery of Devalua was subsequently confirmed in many laboratories around the world.

Other brain tissue, if necessary, takes part in the electrical interaction between brain nerve cells, or neurons. Neurons have tree branches, and when the electrical signal reaches the end of one such branch, it propagates further in the form of waves, exactly as we observe on the surface of water. Since neurons are closely adjacent to each other, divergent electrical waves are constantly superimposed on each other. Neural holograms are created that are multiple and subtle in nature. Waves can create an endless kaleidoscopic series of interference paintings in which the adaptability of the brain is rooted on the principle of holography. The holographic principle invariably appears in the wave nature of the interaction of brain nerve cells.

The experiments of academician N.P. Bekhtereva showed that brain activity is performed in accordance with quantum laws. They strongly confirmed that the human brain is an organ that generates wave structures that are adequate to forms of the outside world. Based on these studies, physiologist A.N. Lebedev put forward "an assumption about recording perceived information and storing it in memory in the form of stable holographic patterns formed by different phases of coherent non-extinguishing waves of neural activity that appear in different places in the brain. The waves themselves are a combination of different frequency oscillations, wherein waves of the same frequency can differ in phases and amplitudes. The packet of waves of the same frequency with different phases, scientists began to consider the simplest unit of memory.

The source of the holographic recording is wave processes and pulses arising during the operation of nerve cells, while information is encoded on a plurality of neurons interacting with each other. The holographic model perfectly describes the properties of information distribution in brain neural networks. Currently, there is no 
method that shows the distribution of information at any point in the information store with such clarity and certainty as the mathematical apparatus of holography does.

According to Professor Bergson's hypothesis: "The nervous system and, above all, the brain" extinguishes "most of the sensory stimuli at the entrance of individual consciousness. Moreover, only information that requires close attention and response is selected from them.

The holographic principle also helps explain why people can determine the source of sound without turning their heads. A hologram is a virtual image that has emerged where it does not exist. Our internal speech senses indicate the presence of this virtual image.

Functional magnetic resonance imaging technologies are a powerful tool that allows you to achieve high-quality fixation of the hologram of the activity of internal speech of the human brain. The introduction of FMRI technologies in various areas of human activity - neuro marketing, professional casting, evaluation of the effectiveness of educational programs, "detection" of lies, etc., will have a huge impact on the further development of mental neurointerfaces.

\subsection{Functional Modes of Mental Neurointerfaces}

Provision of resonant communication of specialists through mental neurointerfaces is carried out using the following modes: request for dialogue, readiness for dialogue, mode of transmission of internal speech, confirmation of mental communication, mode of testing of mental communication.

A dialogue request is made to check the readiness for communication. The mode is activated by interlocutors-inductors before the beginning of mental communication.

The mode of readiness for dialogue was introduced to confirm the consent of the recipient interlocutor to mental communication.

The internal speech mode of the inductor is activated after receiving a signal from the recipient of readiness for mental communication. In this mode, magnetic resonance hologram of internal speech of inductor is transmitted to recipient.

The mental communication confirmation mode is activated after activation of brain activity of the recipient by magnetic resonance hologram of inductor. This mode confirms the activation of the recipient's internal speech.

The mental communication test mode is entered to configure it. It is turned on to check the similarity of the internal speech of the inductor and the recipient. The check is carried out using texts.

After setting up mental communication, the interlocutors will activate the dialogue with internal speech.

\section{Nanoresonators}

Nanoresonators consists of two comb-like intersecting sound or photon-crystal waveguides at the point of intersection forming resonance chamber. Slots are made in zone of resonant chamber, at that length of slot is not less than 2 times its width. Slots can be located at equal distance from center of intersection of resonant chamber diagonals. In addition, the slits may be disposed within the waveguide and may also be filled with a nonlinear optical material such as chalcogenide glass. Optical or sound transistors serve to create buffer elements that can store information directly in optical or audio form for several seconds. The nanoresonator can be calculated for different wavelength ranges. For example, for the wave range used in telecommunications (1.30-1.65 $\mu \mathrm{m})$. It may also be an optical wavelength range $(0.39-0.75 \mu \mathrm{m})$ or an audio range. Simple geometry of nanoresonators makes it possible to manufacture them by high-tech means (Asadi et al., 2020).

The sound resonator amplifies the hologram of internal speech. The amplified hologram is transmitted by the mental interface to the fiber optic network.

\section{Fiber Optic Network}

Fiber-optic lines can be used to transmit magnetic resonance holograms in the optical range. Fiber-optic communication lines are specialized communication lines over which information is transmitted by a light beam in a certain encoding. This technology is primarily used to transmit data in local and global networks over a sufficiently long distance. In such networks, fiber is used. and data is transmitted by a beam of light. The sending device encodes the information as packets. Then the data is translated into the format that can be transmitted using light. Almost instantly, the data reaches the receiver and is translated into a format understandable to the recipient. Depending on the type of active equipment used, the transfer rate can be tens of gigabytes and even terabytes per second. 
In addition to unique throughput characteristics, fiber-optic communication lines have another valuable property low losses and insensitivity to electromagnetic fields. They allow you to combine jobs at any distance in various areas of activity (Bryndin, 2020b). Well-scale demonstration of distributed pressure sensing using fiber-optic DAS and DTS (Ekechukwu \& Sharma, 2021). Investigation of the heat distribution in dry friction systems during fade and recovery using fiber-optic sensing and infrared technology (Albers, Klotz, Fink, \& Ott, 2021). Easychair preprint performance of effect for XPM and FWM in fiber optics (Abood \& Hamdi, 2021). Blood glucose measurement using fiber optics for non-invasive device (Sandesh \& Vipul, 2021). Modified structure of photonic crystal fiber with dispersion and confinement loss properties for wideband applications (Bashir, 2021). A survey on integrated access and backhaul networks (Zhang, Kishk, \& Alouini, 2021). Uncertainties in measuring soil moisture content with actively heated fiber-optic distributed temperature sensing ( $\mathrm{Wu}$, Lamontagne-Hallé, \& McKenzie, 2021). A High-dynamic-range digital RF-Over-Fiber Link for MRI receive coils using delta-sigma modulation (Fan et al., 2021). christoph füllner. KIGLIS: smart networks for smart cities (Bogdoll et al., 2021). Breakthrough or heresy fiber optic cleaning without inspection (Edward, 2021). Investigation of the possibilities of developing an aircraft data system based on fiber optic sensors (Minaxanım \& İslam, 2021).

Due to the low attenuation of the light signal, optical systems can combine work areas at significant distances (more than $100 \mathrm{~km}$ ) without the use of additional repeaters (amplifiers). Fibre-optic communication provides reliable protection against unauthorized access and interception of confidential information. This ability of optics is due to the lack of radiation in the radio range, as well as high sensitivity to oscillations. In case of wiretapping attempts, the built-in monitoring system can turn off the channel and warn of a suspected hack. The use of fibre-optic communication lines allows you to easily increase the computing capabilities of local area networks by installing faster active equipment, without replacing communications.

\section{Conclusion}

The basis of natural phenomena is resonance, as the process of its stability, as well as the law of gold section. as a measure of harmony. On these two basic natural foundations, the resonant technology of mental communication of people with neurointerfaces is built.

The proposed technology of resonant communication with internal speech by mental neurointerfaces with two-way communication through fiber optic communication using multi-channel wave nano resonators requires additional interdisciplinary research by specialists in related professions to effectively combine different technologies and make widespread use of thought dialogue for practical purposes.

The resonant technology of mental communication of people with neurointerfaces ensures the secrecy and effectiveness of dialogue and can be used in various spheres of life. Mental neurointerfaces will have many areas of use and a huge spread among specialists in various professions and even among the population for intimate dialogue. When the mental neurointerface is revealed all opportunities, then they will be used, if not all, then most, while all spheres of society will undergo changes. It is worth morally and technologically preparing for such changes and knowing at least the principles of work, so that when the time comes not to be left behind everyone.

Mental neurointerfaces are perhaps one of the most fantastic technologies, the development of which brings us closer to a new, unlike today, world of the future. The resonant technology of mental communication of people with neurointerfaces is potentially very powerful technology, it can significantly change not only human society, but also the person himself. A common understanding will become a criterion for communication. Empathy will help us accurately predict the behavior of other people to communicate and work with themselves like that. In an experiment conducted by Charles Tarth, professor of physiology at the University of California, the answer was: "What happens if two or more people try to design the same imaginary reality - so that their mental waves are superimposed so that a hologram of a new world arises?" Tart found two high school students, Ann and Bill, who could enter a deep trance, and were also quite skillful hypnotists themselves. He got Anne to hypnotize Bill, and Bill to hypnotize Anne in that state. Tart suggested that thanks to such an unusual procedure, the connection between them should be strengthened. And he was right. When in this mutual hypnotic state they opened their eyes, in a matter of seconds both were on the shore.

Despite all the favorable conditions for the development of neurointerfaces, the probability that they will reach such heights is quite high, but not equal to $100 \%$. There is always a chance of unforeseen circumstances. For example, researchers and engineers will not be able to overcome the existing difficulties in implementation, because of which the technology will cease to develop or if the device is too expensive for widespread use. And yet, given all the factors, the probability of such an outcome is very unlikely, the maximum development will be delayed for a couple of decades. So all we have to do is wait or make our own contribution to the development and spread of this technological miracle. 
The development of resonant communication technology in the future can contribute to the transfer of holographic information of semantic memory of one person to another person. On the basis of numerous experiments performed at the Institute of Clinical and Experimental Medicine at the Siberian Branch of the Russian Academy of Sciences, Academician Treasurer came to the conclusion: "A living creature (soul) first designs itself in the form of a holographic field image and on the basis of this particular image builds its own, specific earthly biochemical body." Academician of the Russian Academy of Sciences Garyaev and his colleagues experimentally proved that such a hologram arises even before the birth of a holistic organism. Roughly speaking, information coming from the outside in relation to the embryo causes its chromosomes to create a certain wave image. "This image is a hologram and dictates to dividing cells when and where the legs, hands, head should grow. The wave image is filled with matter just as the mold is filled with casting, "writes Garyaev. On the principles of photodynamics, it will be possible to transfer a holographic copy of a person to a biorobot.

\section{References}

Abood, M. S., \& Hamdi, M. (2021). EasyChair Preprint Performance of Effect for XPM and FWM in Fiber Optics. Conference: International Conference on Emerging Technologies and Intelligent Systems (ICETIS 2021). Malaysia.

Albers, A., Klotz, T., Fink, C., \& Ott, S. (2021). Investigation of the heat distribution in dry friction systems during fade and recovery using fiber-optic sensing and infrared technology. Friction, 1-14. https://doi.org/10.1007/s40544-021-0506-4

Asadi, N., Wang, Y., Olson, I., \& Obradovic, Z. (2020). A heuristic information cluster search approach for precise functional brain mapping. Human brain mapping, 41(9), 2263-2280. https://doi.org/10.1002/hbm.24944

Bashir, K. (2021). Modified Structure of Photonic Crystal Fiber with Dispersion and Confinement Loss Properties for Wideband Applications. Journal of Independent Studies and Research Computing, 17(2).

Bogdoll, D., Matalla, P., Füllner, C., Raack, C., Li, S., Käfer, T., ... \& Bonk, R. (2021). KIGLIS: Smart Networks for Smart Cities. arXiv preprint arXiv:2106.04549. https://doi.org/10.1109/ISC253183.2021.9562826

Bryndin, E. (2020a). Transformation Modeling of Nanostructures by Cognitive Systems according to Big Smart Data. Chepter 3, Pages: 21-28. / Book "New Materials: Preparation, Properties and Applications in the Aspect of Nanotechnology" (P. 250). USA: Nova Science Publisher.

Bryndin, E. (2020b). Implementation of international telemedicine network with rapid coronavirus registration by resonant technology to neutralize the pandemic. Computational Biology and Bioinformatics, 8(2), 29-35. https://doi.org/10.11648/j.cbb.20200802.11

Bryndin, E. (2021). Mental Communication of Internal Speech with Communicative Associative Robot via Spectral Neurointerface. American Journal of Applied Psychology, 10(3), 55-64. https://doi.org/10.11648/j.ajap.20211003.11

Bryndin, E. G. (2018). Psychological and social aspects formations of thinking, consciousness and behavior. SM Physical Medicine \& Rehabilitation, 2(1), 1-5.

Duff, M. C., Covington, N. V., Hilverman, C., \& Cohen, N. J. (2020). Semantic memory and the hippocampus: revisiting, reaffirming, and extending the reach of their critical relationship. Frontiers in Human Neuroscience, 13, 471. https://doi.org/10.3389/fnhum.2019.00471

Edward, F. (2021). Breakthrough or Heresy Fiber Optic Cleaning without Inspection.

Ekechukwu, G. K., \& Sharma, J. (2021). Well-scale demonstration of distributed pressure sensing using fiber-optic DAS and DTS. Scientific reports, 11(1), 1-18. https://doi.org/10.1038/s41598-021-91916-7

Fan, M., Brown, R. W., Gao, X., Mandal, S., Petropoulos, L., Yang, X., ... \& Fujita, H. (2021). A high-dynamic-range digital RF-over-fiber link for MRI receive coils using delta-sigma modulation. Review of Scientific Instruments, 92(6), 064708.

Ilya, S. B., \& Alexandra, P. (2020). Transcranial magnetic stimulation in cognitive neuroscience: Methodological basis and safety. Российский журнал когнитивной науки, 7(3), 25-44. https://doi.org/10.47010/20.3.2

Liu, L., Yan, X., Li, H., Gao, D., \& Ding, G. (2020). Identifying a supramodal language network in human brain with individual fingerprint. NeuroImage, 220, 117131. https://doi.org/10.1016/j.neuroimage.2020.117131

Minaxanım, A., \& İslam, İ. (2021). Investigation of the possibilities of developing an aircraft data system based on fiber optic sensors. Conference: Fevral məruzələri 2021, Bak1 
Mitsuhashi, T., Sonoda, M., Jeong, J. W., Silverstein, B. H., Iwaki, H., Luat, A. F., ... \& Asano, E. (2021). Four-dimensional tractography animates propagations of neural activation via distinct interhemispheric pathways. Clinical Neurophysiology, 132(2), 520-529. https://doi.org/10.1016/j.clinph.2020.11.030

Sandesh, T., \& Vipul, S. (2021). Blood Glucose Measurement using Fiber Optics for Non-Invasive Device. Conference: 21st International Conference on Science, Engineering and Technology. Vellore. 2021

Toropov, N. A., \& Sumetsky, M. (2016). Permanent matching of coupled optical bottle resonators with better than $0.16 \mathrm{GHz}$ precision. Optics letters, 41(10), 2278-2281. https://doi.org/10.1364/OL.41.002278

Wang, X., Liang, X., Jiang, Z., Nguchu, B. A., Zhou, Y., Wang, Y., ... \& Qiu, B. (2020). Decoding and mapping task states of the human brain via deep learning. Human brain mapping, 41(6), 1505-1519. https://doi.org/10.1002/hbm.24891

Wu, R., Lamontagne-Hallé, P., \& McKenzie, J. M. (2021). Uncertainties in Measuring Soil Moisture Content with Actively Heated Fiber-Optic Distributed Temperature Sensing. Sensors, 21(11), 3723. https://doi.org/10.3390/s21113723

Zhang, Y., Kishk, M. A., \& Alouini, M. S. (2021). A survey on integrated access and backhaul networks. arXiv preprint arXiv:2101.01286. https://doi.org/10.3389/frcmn.2021.647284

\section{Copyrights}

Copyright for this article is retained by the author(s), with first publication rights granted to the journal.

This is an open-access article distributed under the terms and conditions of the Creative Commons Attribution license (http://creativecommons.org/licenses/by/4.0/). 